\title{
Morfometría del Cuerpo Calloso y de los Cuerpos Mamilares en el Alcoholismo por Resonancia Magnética
}

\author{
Morphometry of the Corpus Callosum and Mammillary Bodies in Alcoholism using Magnetic \\ Resonance
}

Elba Beatriz Tornese* \& Martín Javier Mazzoglio y Nabar**

TORNESE E. B. \& MAZZOGLIO Y NABAR, M. J. Morfometría del cuerpo calloso y de los cuerpos mamilares en el alcoholismo por resonancia magnética. Int. J. Morphol., 31(4):1233-1242, 2013.

RESUMEN: Las alteraciones morfológicas encefálicas en individuos que padecen alcoholismo crónico explican los severos trastornos neurocognitivos, principalmente amnésicos anterógrados, y neuropsiquiátricos debido al carácter neurotóxico y déficits nutricionales que genera. El objetivo fue describir la morfología del cuerpo calloso y los cuerpos mamilares en dependientes al alcohol y su relación volumétrica otras áreas cerebrales. Se estudiaron morfológica y morfométricamente con resonancia magnética los cerebros de 26 masculinos, edades 29 a 51 años, con diagnóstico de trastorno por consumo de alcohol tipo dependencia (DSM IV). Fueron criterios de exclusión: patologías hepáticas, autoinmunes, neurológicas centrales y antecedentes neurológicos no relacionados con el consumo, traumatismo craneoencefálico o neurocirugías previas. Se cuantificó la volumetría (método ROI) de: corteza prefrontal, hipocampo, cuerpo amigdalino, tálamo y cuerpos mamilares; y la morfometría bidimensional de las regiones del cuerpo calloso (métodos de Witelson y de Clarke). El procedimiento fue sometido a pruebas estadísticas y cumplió con los reparos ético-legales vigentes. Todos los volúmenes de pacientes dependientes fueron menores. Se registró según severidad reducción volumétrica significativa en corteza prefrontal, cuerpo mamilar, hipocampo y tálamo izquierdos, seguidos por el cuerpo amigdalino derecho. La superficie con mayor afectación del cuerpo calloso fue el 1/3 anterior. Se determinaron correlaciones entre la afectación de los cuerpos mamilares y el cuerpo calloso con estructuras subcorticales. El compromiso del cuerpo calloso y los cuerpos mamilares presentó correlación con la afectación del hipocampo, cuerpo amigdalino y tálamo, y una estrecha relación con el tiempo y cantidad de consumo. Estas alteraciones morfométricas son indicadores de severidad cognitiva y alteración comportamental.

PALABRAS CLAVE: Morfometría; Cuerpo calloso; Cuerpos mamilares; Alcoholismo.

\section{INTRODUCCIÓN}

Se ha determinado que las alteraciones morfológicas corticales cerebrales (frontales e hipocampales) y subcorticales (diencefálicas) explican los severos trastornos cognitivos en consumidores de alcohol.

Estudios preliminares e iniciales reportaron el aumento ventricular relacionado con la reducción volumétrica de las cortezas del cerebro, especialmente la prefrontal, pero el alcohol genera alteraciones encefálicas variadas y variables debido a su carácter neurotóxico, a los déficits nutricionales y a las características individuales de los sujetos dependientes, determinando diversas manifestaciones clínicas (Aboitiz et al., 1992a, 1992b; Bilir et al., 1998; Clarke et al., 1989; de Lacoste et al., 1985; Estruch et al., 1997; Fein et al., 2002).
El objetivo del presente trabajo de investigación fue describir la morfología del cuerpo calloso y los cuerpos mamilares en sujetos dependientes al alcohol, determinando su implicancia y su relación con la volumetría de áreas cerebrales seleccionadas y características de la dependencia.

\section{MATERIAL Y MÉTODO}

Se estudiaron 26 pacientes de sexo masculino, de edades entre 29 a 51 años (promedio $=37,67, \mathrm{DE}=6,81$ ), con diagnóstico de trastorno por consumo de alcohol tipo dependencia (F10.2x) con remisión parcial temprana, según

Médica (UBA). Jefa de Servicio del Hospital Neuropsiquiátrico “Dr. Braulio A. Moyano”. Doctora en Medicina, Médica Psiquiatra y Médica Legista de la UBA. Profesora Titular de la Universidad Abierta Interamericana. Profesora Adjunta Equiparada de Anatomía y Docente Autorizada del Departamento de Salud Mental, Facultad de Medicina, Universidad de Buenos Aires, Buenos Aires, Argentina.

** Médico (UBA). Especialista en Psiquiatría. Docente Adscripto de Anatomía y de Farmacología, III Cátedra de Anatomía y III Cátedra de Farmacología, Facultad de Medicina, UBA. Docente Auxiliar de Psiquiatría, Universidad de Buenos Aires, Buenos Aires, Argentina. 
parámetros del DSM IV. Según historial clínico, en promedio presentaron 9,7 años de consumo de alcohol ( $\mathrm{DE}=3,93)$, 6,35 l/d ( $\mathrm{DE}=2,33)$; sólo 5 participantes $(19,26 \%)$ manifestaron ser monoconsumidores y el resto consumidores de cerveza, vino y bebidas blancas.

Se establecieron como criterios de exclusión: insuficiencia hepática o patología hepática descompensada o severa (por cirrosis entre otras), diabetes, vasculopatías, actuales patologías neurológicas centrales, antecedentes de patología neurológica no relacionada con el consumo (encefalitis, epilepsia), de traumatismo craneoencefálico o neurocirugías previas.

Dado que los cambios cerebrales (bioquímicos como posteriormente perfusionales y morfológicos) y los déficits cognitivos son parcialmente secundarios al daño hepático en pacientes dependientes con cirrosis alcohólica, se incluyeron sujetos dependientes detoxificados siendo dicho parámetro determinado por análisis bioquímicos (factores de coagulación y enzimas hepáticas) y ecográficos.

El grupo control estuvo comprendido por 25 pacientes de sexo masculino, con edades entre 30 a 50 años (promedio=38,27, $\mathrm{DE}=6,56$ ), sin diagnóstico de patología neuropsiquiátrica ni antecedentes de traumatismo craneoencefálico, neurocirugías, patologías orgánicas ni tratamientos neuropsicofarmacológicos en curso.

Se tomaron como reparos éticos para la presente investigación: requerimiento del consentimiento informado previo a la realización de las neuroimágenes, reserva de la identidad de los participantes y cumplimiento de los requisitos estipulados por las GCP (Good Clinical Practice), Disposición ANMAT 6677/10 y principios éticos con origen en la Declaración de Helsinski.

Previo a la realización de las neuroimágenes se realizaron pruebas bioquímicas en sangre, la Prueba de Dominancia Manual (Inventario de Edimburgo) y tests neuropsicológicos.

En ambos grupos se estudiaron morfológica y morfométricamente los encéfalos por Resonancia Magnética (RM) sin contraste con cuantificación volumétrica. Se utilizó un Resonador magnético de 1.5T, se exploraron las regiones predeterminadas en secuencias T1, T2 y FLAIR, con registro en planos axial, coronal y sagital. Las imágenes en T1 fueron realizadas mediante cortes de $1,5 \mathrm{~mm}$ en el plano axial y secuencias de $1,5 \mathrm{~mm}$ y $3 \mathrm{~mm}$ en el plano coronal perpendiculares al eje del plano temporal y del eje mayor encefálico, las que luego fueron obtenidas por gradiente de 3D con extracción de artificios y reconstruc- ción de imágenes estáticas firmes. La cuantificación de estructuras fue llevada a cabo por un análisis de procesamiento semiautomático con la opción region of interest (ROI), se corrigieron manualmente y se determinaron los volúmenes en ambos hemisferios de: corteza prefrontal (CPF), hipocampo $(\mathrm{H})$, cuerpo amigdalino $(\mathrm{CA})$, tálamo (T) y cuerpos mamilares $(\mathrm{CM})$; como también la morfometría bidimensional de las subregiones del cuerpo calloso (CC). A los participantes se les indicó abstenerse de ingerir lorazepam u otra medicación sedativa por el término de 15 días anteriores a los estudios imagenológicos.

La cuantificación volumétrica de la corteza prefrontal se obtuvo según parámetros descriptos por Wible et al. (1995), y la correspondiente al hipocampo y cuerpo amigdalino se realizó sobre cortes coronales a $3 \mathrm{~mm}$ perpendiculares al eje del plano temporal. En el caso del $\mathrm{H}$, acorde con los trabajos de normatización y las delimitaciones morfométricas de Pruessner et al. (2000), sus medidas incluyen el hipocampo, el giro dentado, el alveus, la fimbria y el subiculum.

La volumetría del tálamo se obtuvo mediante su desconexión imagenológica a través de la masa intermedia y los reparos anatómicos incluyeron lateralmente a la cápsula interna, superiormente las fibras peritalámicas, el borde posterior se demarcó mostrando los fórnix; los cuerpos geniculados fueron excluidos.

Para la cuantificación de los cuerpos mamilares se utilizaron entre 4 y 6 imágenes en T1. Dichos núcleos son estructuras esféricas de aproximadamente $5 \mathrm{~mm}$ de diámetro y están compuestos por 2 núcleos (medial y lateral). El núcleo medial compone el $90 \%$ del volumen de la estructura (Bilir et al.) y su demarcación se realiza en cortes de 1,5 $\mathrm{mm}$ dado que aparece bordeado por una cápsula fibrosa atenuada. El límite inferior es marcado por una señal de intensidad que emerge desde la fosa interpeduncular, junto a una línea lateral por la sustancia nigra. El núcleo mamilar lateral yace ventral y lateral al mamilar medial y dado su pequeño tamaño es incluido en la medición volumétrica aunque no se lo distinga visualmente.

La superficie del cuerpo calloso, desde el rostrum o cabeza hasta el esplenio, se mide sobre el corte sagital potenciado en $\mathrm{T} 1$ que mejor representa la región sagital media (Iai et al., 1994; Laissy et al., 1993; Verger et al., 2001). Los criterios para determinar la orientación sagital media fueron establecidos por Giedd et al. (1996). Una vez determinado el corte sagital medio se selecciona el cuerpo calloso como la ROI y mediante la digitalización se crea automáticamente un contorno sobre la ROI, la que será calculada automáticamente por el programa. Se examinaron 2 
diferentes métodos para la subdivisión del CC en áreas de interés anatomo-clínico. El primer método, adaptado por Witelson (1989), propone subdivisiones basadas en fracciones matemáticas sobre el máximo de la distancia ántero-posterior del cuerpo calloso (Fig. 1). El segundo método, adaptado de Clarke et al., utiliza una línea curva a modo de referencia dentro del cuerpo calloso (Fig. 2). En ambos casos, el cuerpo calloso es subdividido en la mitad, en tercios y un quinto. El resultado de las 5 subdivisiones, de anterior a posterior, es: tercio anterior (incluye el rostrum o pico, genu o rodilla y cuerpo anterior), mitad anterior del cuerpo, mitad posterior del cuerpo, istmo o isthmus y esplenio o splenium. Sumado a las áreas del CC, se cuantifican dos líneas (Fig. 2): ancho máximo del esplenio y ancho mínimo del cuerpo.

El esquema de parcelación descripto por Witelson fue diseñado sobre la base del estudio con microscopía electrónica de las fibras callosas en el mono, y posteriormente validado por Aboitz et al. (1992a) en un análisis post-mortem y Zaidel et al. (1995) en un análisis in vivo.

Cabe destacar que el área del cuerpo calloso en plano mediosagital no correlaciona significativamente con el área cerebral en plano mediosagital. Dada dicha particularidad, al igual que sucede en los casos de morfometría cerebelar, las medidas del cuerpo calloso no se normatizan según el área cerebral.

Obtenidos los datos se aplicaron pruebas de estadística descriptiva e inferencial (coeficientes $r$ y R2) y se utilizó la prueba T de Student (t-Test) para obtener la significación estadística de los parámetros cuantificados volumétricamente que se definió como $\mathrm{p}<0,01$.

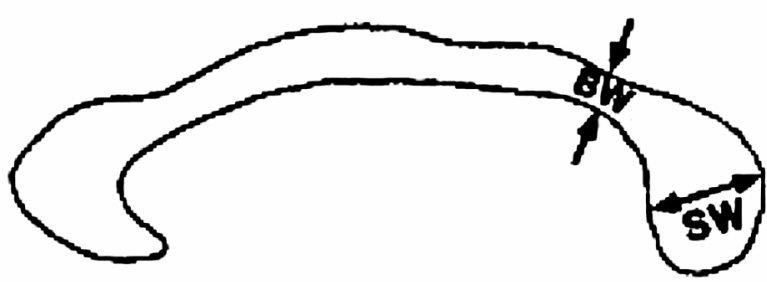

Fig. 2. Cuantificación de ancho máximo del esplenio (SW) y ancho mínimo del cuerpo (BW).

\section{RESULTADOS}

El estudio aplicado para determinar la dominancia manual concluyó que los 22 hombres dependientes, presentaron dominancia manual derecha $(84,61 \%)$, mientras que en controles se determinó la dominancia derecha en el $80,77 \%(\mathrm{n}=21)$.

Todos los volúmenes registrados en dependientes fueron menores. La corteza prefrontal izquierda registró una disminución volumétrica del $23,35 \%$, mientras que la derecha del 19,11\%; ambas diferencias fueron significativas $(\mathrm{p}<0,001)$. El hipocampo izquierdo en dependientes presentó mayor afectación volumétrica siendo su disminución del $12,24 \%$ ( $<<0,001)$; en el derecho fue de 6,27\% ( $<<0,01)$. El tálamo izquierdo registró una atrofia de 8,38\% ( $p<0,001)$, mientras que en su contralateral se cuantificó una reducción de $7,72 \%$ no significativa $(\mathrm{p}<0,1)$. El cuerpo amigdalino presentó mayor disminución volumétrica en el hemisferio derecho $(5,62 \%, \mathrm{p}<0,01)$ que en el izquierdo $(5,38 \%, \mathrm{p}<0,01)$

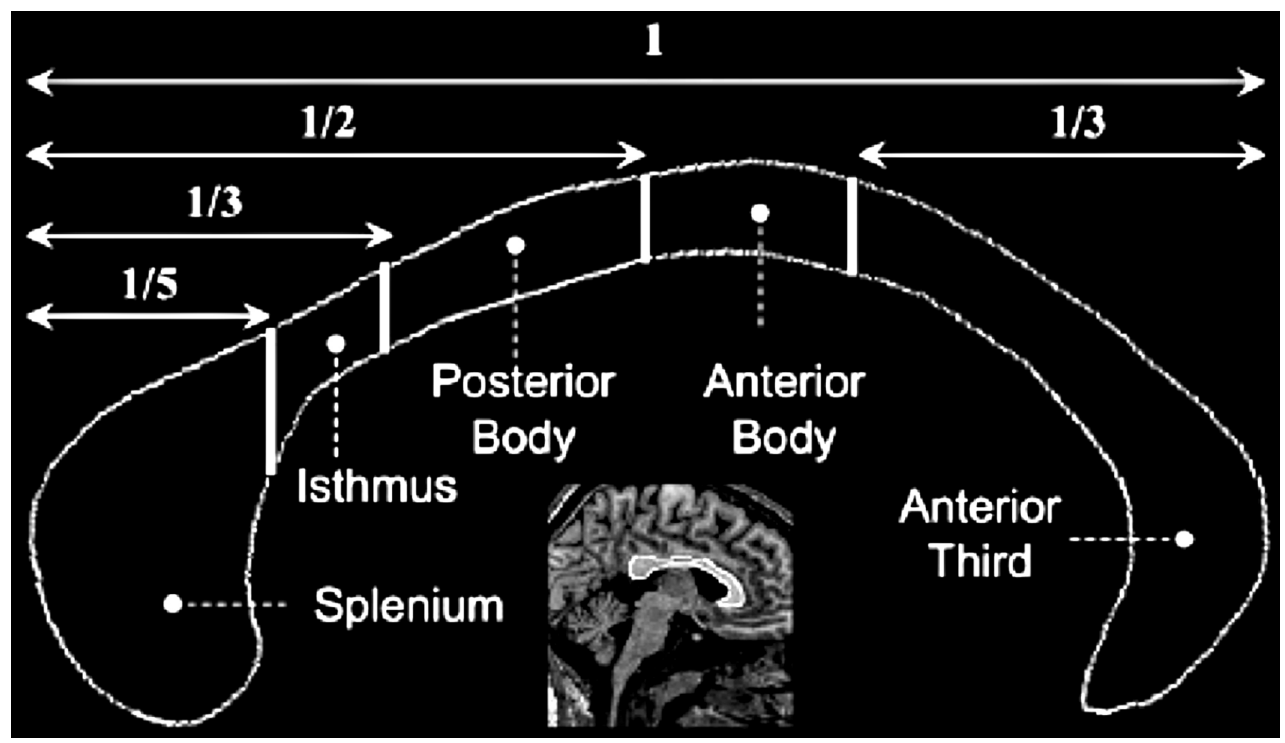

Fig. 1. Cuantificación de superficie del cuerpo calloso y subdivisiones según método de Witelson. 
(Figs. 3 y 4). El cuerpo mamilar izquierdo registró una significativa disminución del 15,43\% (p<0,001), mientras que en el derecho fue de 10,35\% (p<0,01) (Fig. 3).

En relación con la morfometría de superficie del cuerpo calloso, las áreas más afectadas significativamente fueron: $1 / 3$ anterior $(15,98 \%, \mathrm{p}<0,001)$; istmo $(11,05 \%$; $\mathrm{p}<0,001) ; 1 / 2$ anterior del cuerpo $(9,83 \% ; \mathrm{p}<0,01)$ y esplenio $(8,52 \%, \mathrm{p}<0,01)$. Las diferencias registradas en la $1 / 2$ posterior del cuerpo no fueron significativas $(3,83 \%, \mathrm{p}=0,12)$. La longitud del ancho máximo del esplenio registró una disminución en dependientes de 9,29\% ( $\mathrm{p}<0,001)$, mientras que la longitud del ancho mínimo del cuerpo fue 13,68\% menor en dependientes $(\mathrm{p}<0,01)$ (Fig. 4).

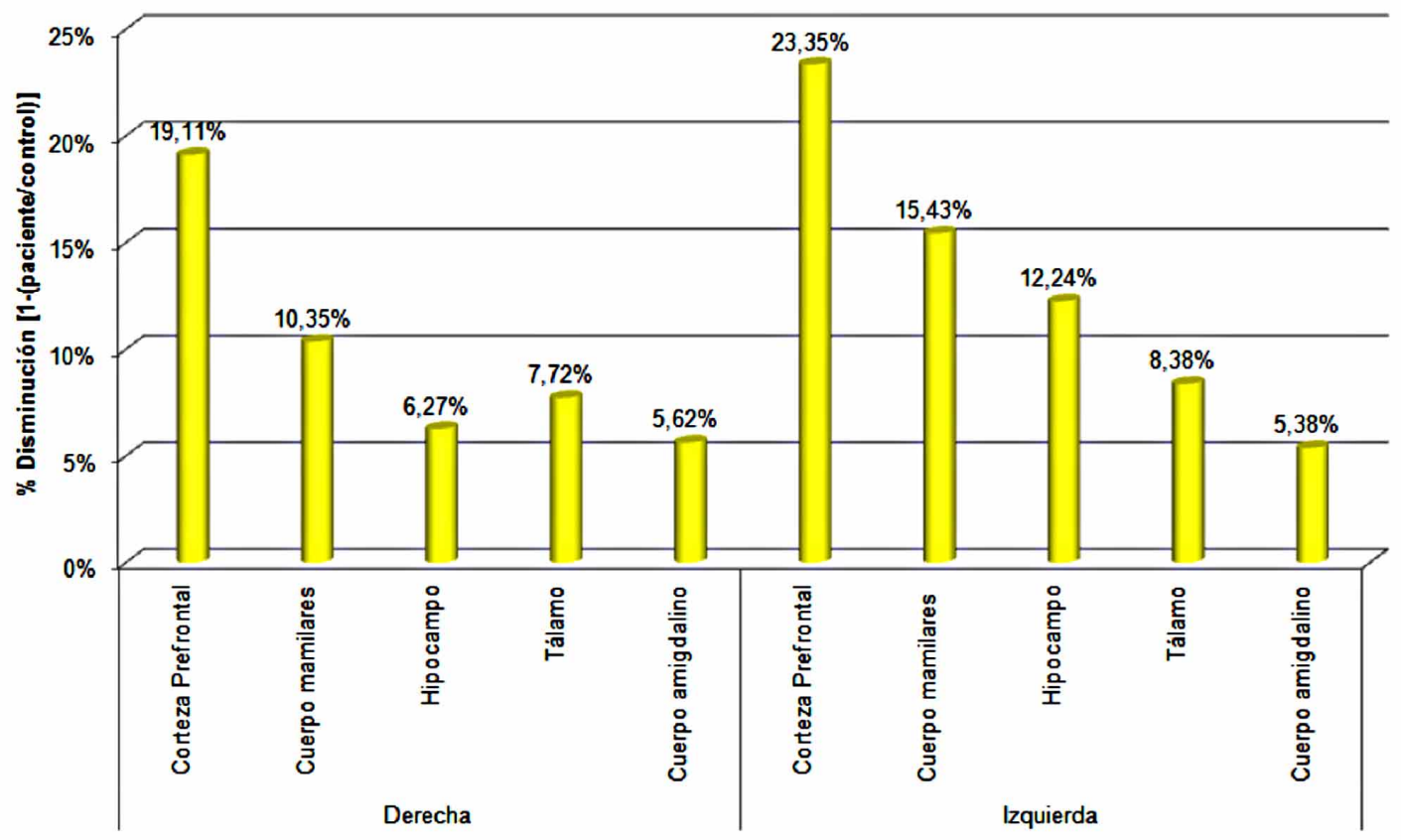

Estructura anatómica

Fig. 3. Porcentaje de disminución volumétrica de estructuras cuantificadas por RM según hemisferio cerebral entre dependientes y controles.

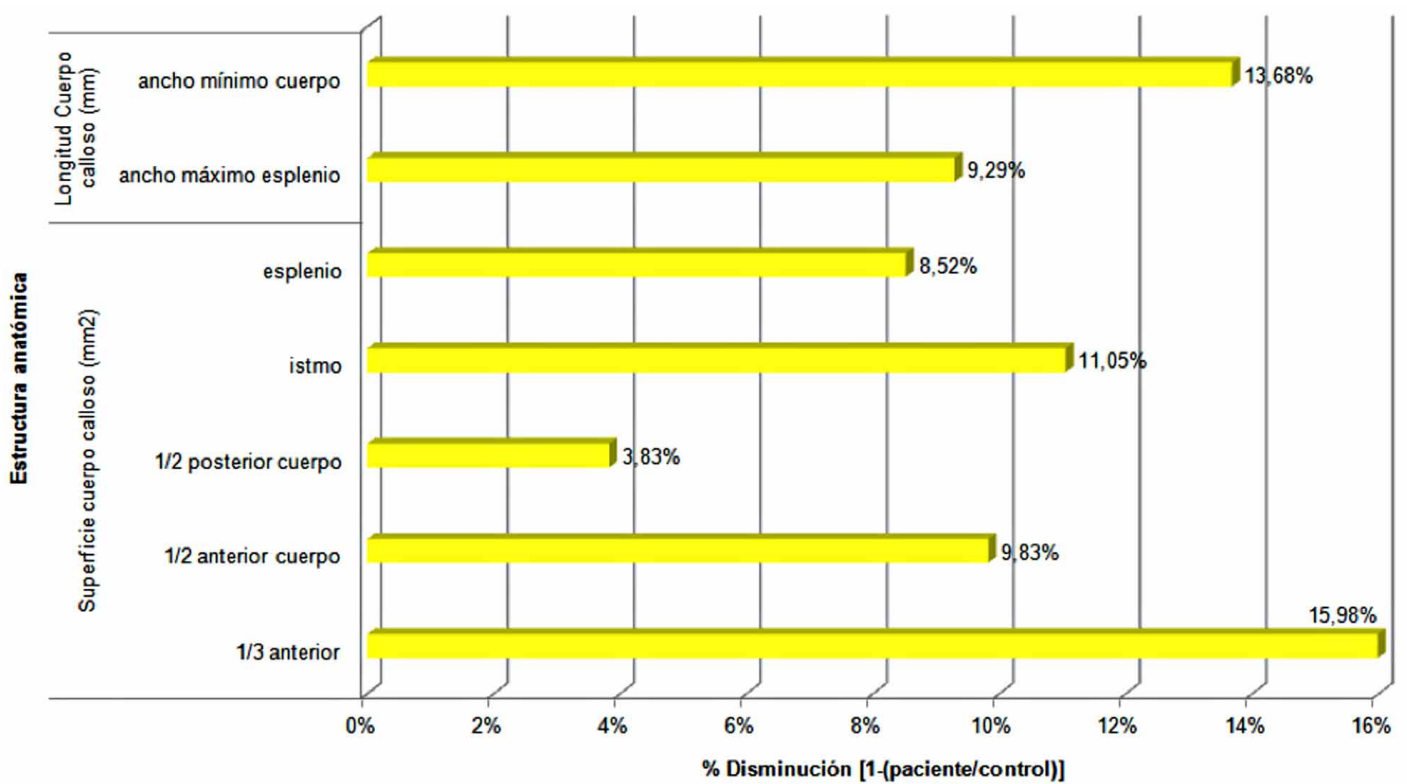

Fig. 4. Porcentaje de disminución de superficies y longitudes cuantificadas por RM según hemisferio cerebral entre dependientes y controles. 
Por gráfico de correlación se determinó que:

- El cuerpo mamilar izquierdo fue la única estructura que presentó correlación negativa significativa con la cantidad de $1 / d\left(r=-0,79 ; R^{2}=0,34\right)$ y registró una correlación positiva e implicancia con la volumetría del cuerpo amigdalino izquierdo ( $\left.r=0,93, \mathrm{R}^{2}=0,88\right)$ (Fig. 5).
- La volumetría del cuerpo mamilar derecho presentó correlación positiva con la morfometría de ambos hipocampos $\left(\right.$ derecho $=r=0,93 ; R^{2}=0,81 ;$ izquierdo $\left.=r=0,88 ; R 2=0,80\right)$ y del tálamo derecho $\left(\mathrm{r}=0,98 ; \mathrm{R}^{2}=0,95\right)$ (Fig. 6);

- La superficie del 1/3 anterior del cuerpo calloso mantuvo una correlación negativa con los años de consumo ( $\mathrm{r}=-0,98$;

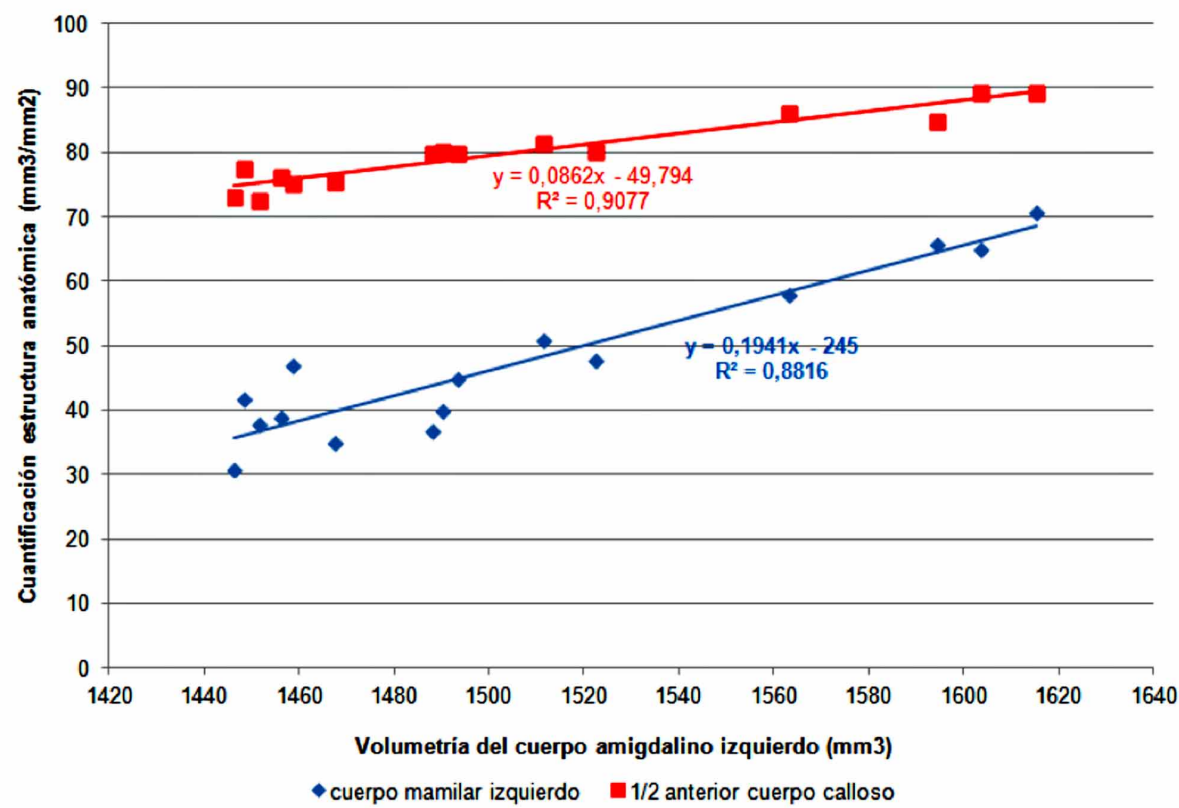

Fig. 5. Volúmenes de cuerpos amigdalinos izquierdos en correlación con superficie de la 1/2 anterior del cuerpo y la volumetría del cuerpo mamilar izquierdo

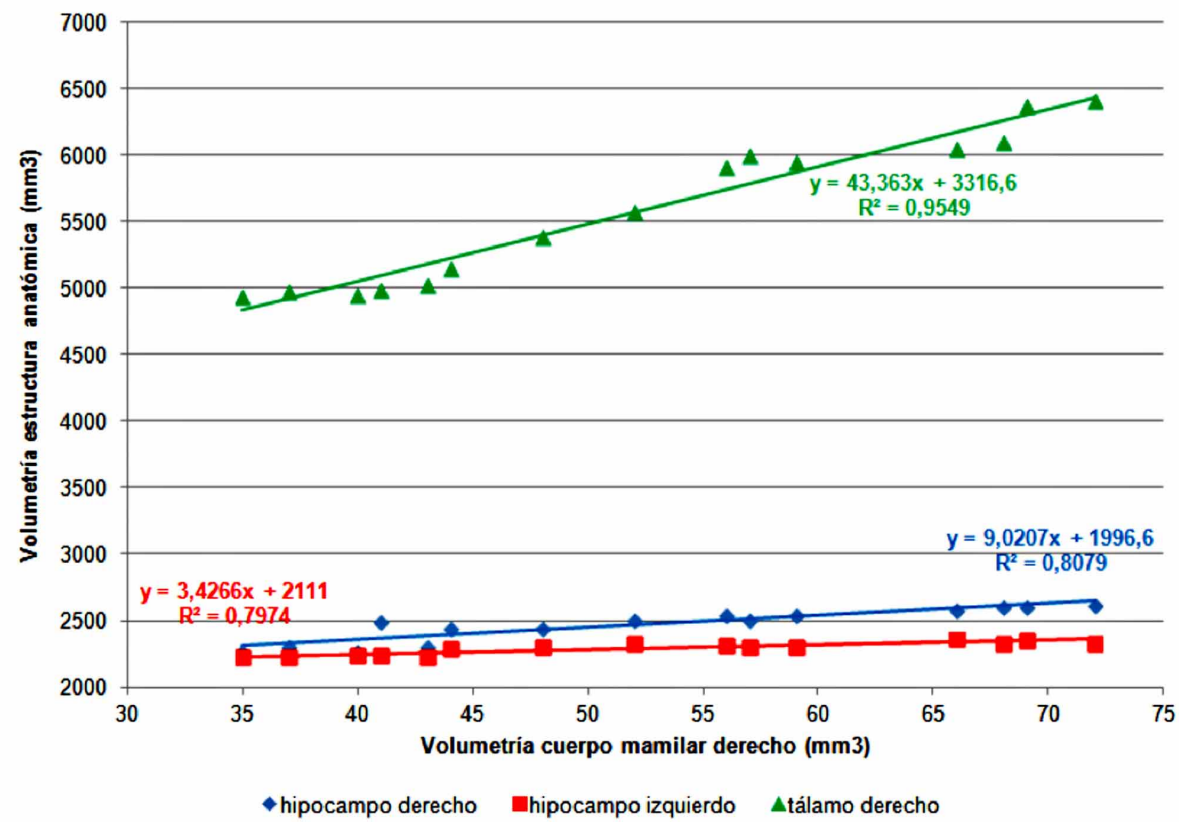

Fig. 6. Volúmenes del cuerpo mamilar derecho en correlación con los correspondientes a los hipocampos de ambos hemisferios y tálamo derecho. 


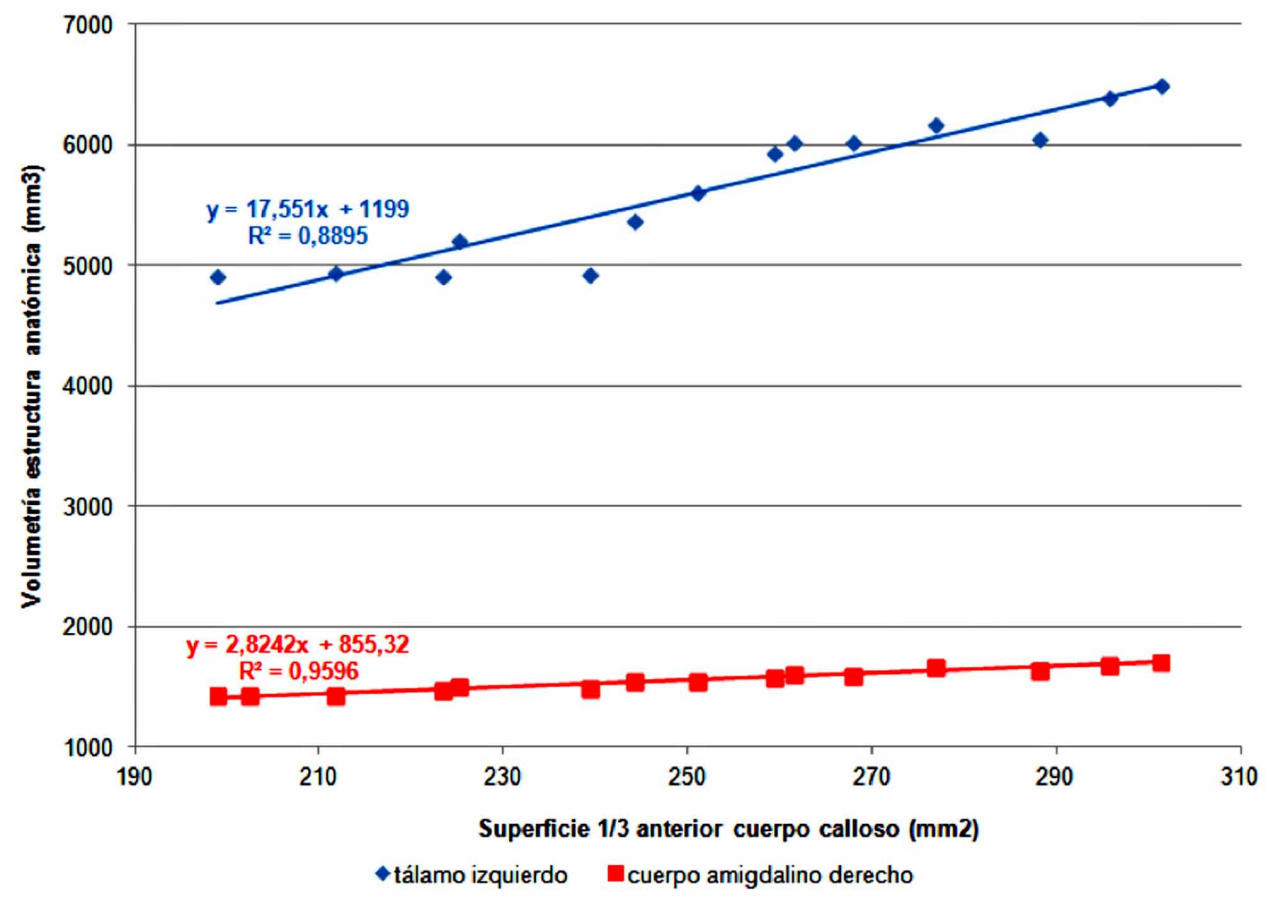

Fig. 7. Superficies del 1/3 anterior del cuerpo calloso en correlación con los volúmenes del tálamo izquierdo y del cuerpo amigdalino derecho.

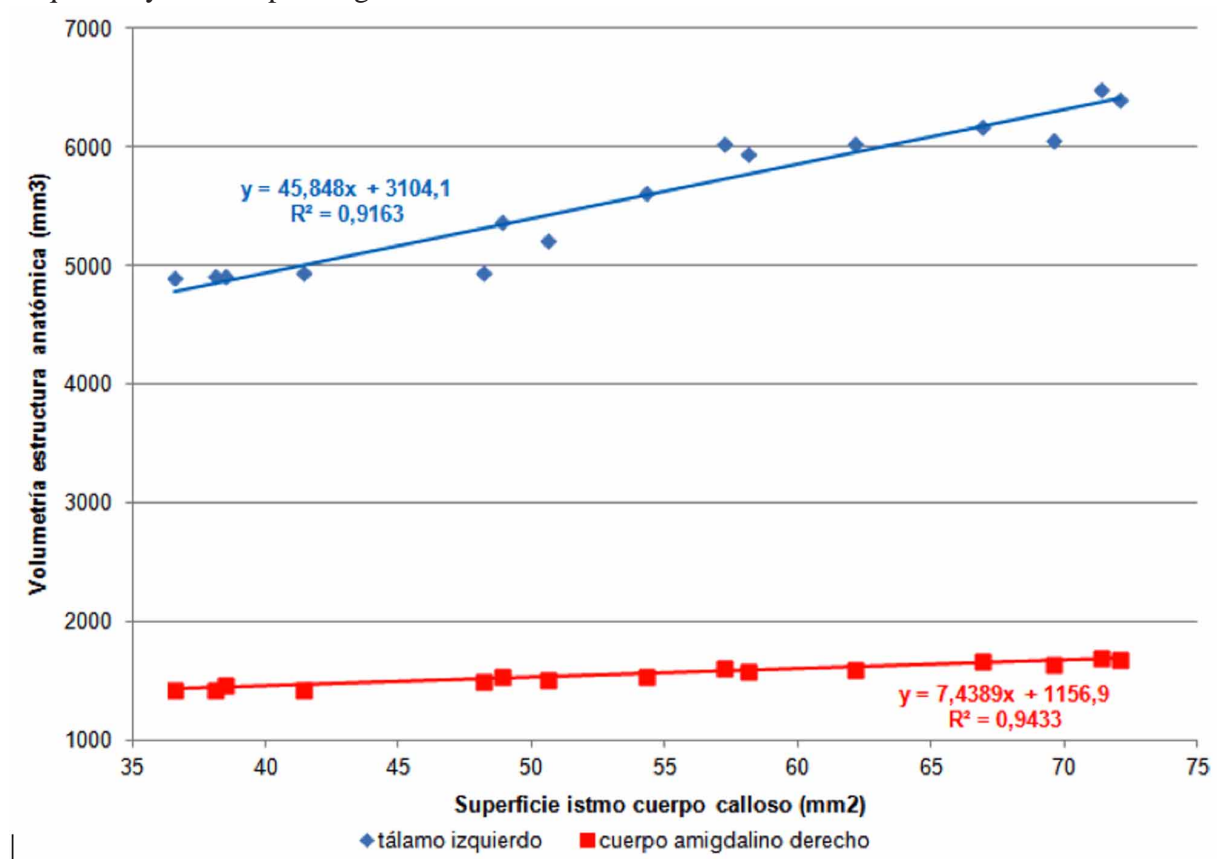

Fig. 8. Superficies del istmo del cuerpo calloso en correlación con los volúmenes del tálamo izquierdo y del cuerpo amigdalino derecho.

$\mathrm{R} 2=0,96)$, y positiva con la volumetría del tálamo izquierdo $(r=0,95 ; \mathrm{R} 2=0,89)$ y cuerpo amigdalino derecho $\left(r=0,98, R^{2}=0,96\right)$ (Fig. 7). En la superficie del istmo se registraron correlaciones con las mismas estructuras pero con magnitudes estadísticas similares $\left(r=-0,98, R^{2}=0,86 ; r=0,96\right.$,
$\mathrm{R}^{2}=0,92$ y $\mathrm{r}=0,97, \mathrm{R}^{2}=0,94$, respectivamente) (Fig. 8);

. La superficie de la 1/2 anterior del cuerpo calloso registró una correlación positiva con la morfometría del cuerpo amigdalino izquierdo $\left(r=0,95, R^{2}=0,91\right)$ (Fig. 5). 


\section{DISCUSIÓN}

Se vio que el alcoholismo tiene la característica de seguir un curso dinámico desde la adicción hasta el mantenimiento de la dependencia, detenido con la abstinencia y recuperado con la recaída en la adicción. Los cambios en la estructura del cerebro acompañan estas fases del curso del alcoholismo.

Estudios previos con neuroimágenes cerebrales estructurales, compararon alcohólicos crónicos con controles sin dependencia al alcohol y demostraron consistentemente un aumento del líquido cefalorraquídeo en los cerebros de los alcohólicos, especialmente en los espacios subaracnoideos. Dichos cambios eran sustentados por los reportes de estudios neuropatológicos que describían una reducción en el peso y el volumen cerebral de los alcohólicos, y quizás podían ser un sustento estructural de la injerencia del etanol como agente neurotóxico de un importante número de regiones cerebrales importantes para las funciones cognitivas superiores.

Numerosos estudios in vivo y postmortem han confirmado la significativa pérdida volumétrica que ocurre en los cerebros de alcohólicos crónicos. Estudios neuropatológicos reportan que las alteraciones volumétricas son principalmente debidas a la pérdida de sustancia blanca, aunque muchos otros reportes han descripto una importante pérdida de sustancia gris consecuente con reducción celular por conteo, tamaño celular o procesos neuronales patológicos consecuentes a la toxicidad del etanol.

Las anormalidades volumétricas cerebrales debidas al consumo de alcohol en la sustancia gris y blanca como de los surcos y el agrandamiento ventricular observados por RM son mayores en personas de edad adulta avanzada que en jóvenes y se reportan más significativamente en la quinta década de la vida.

Con el avance de las técnicas cuantitativas para la evaluación del volumen de sustancia gris en las distintas estructuras evaluadas por imágenes de RM fue posible un acercamiento científico acerca del aumento del LCR en alcohólicos. En un primer estudio, se compararon los aumentos de LCR y las reducciones de sustancia gris en 8 alcohólicos con síndrome de Korsakoff, 12 alcohólicos sin síntomas mnésicos y 13 controles sin dependencia. Ambos grupos de alcohólicos tenían una significativa pérdida de sustancia gris cortical y subcortical asociada con los aumentos del LCR, notándose que dichos cambios eran mayores en alcohólicos con trastornos mnésicos. Mien- tras que la reducción de sustancia gris a nivel diencefálico y de la corteza fronto-parietal superior caracterizaban al alcoholismo en sí, los pacientes con síndrome de Korsakoff se distinguían por su inusual pérdida volumétrica en la porción anterior del diencéfalo, de la corteza temporal mesial y de las regiones órbito-frontales. Luego de esta relación demostrada, se describieron señales hiperintensas en la sustancia blanca y correlaciones entre la disfunción cognitiva con índices de afectación volumétrica en las estructuras por imágenes de RM.

Las anormalidades aparecen y dañan la corteza cerebral, la sustancia gris subcortical y la sustancia blanca determinando desviaciones volumétricas y dilatación de estructuras que serán sus espacios llenados con LCR (Fein et al.; Hommer et al., 2001; Pfefferbaum \& Sullivan, 2002). Dichas alteraciones son más prominentes en determinadas regiones que incluyen a los lóbulos frontales (Pfefferbaum et al., 1997), cuerpo calloso (Estruch et al.; Hommer et al., 1996; Pfefferbaum \& Sullivan; Pfefferbaum et al., 1996) e hipocampo (Sullivan et al., 1996). El daño de la primera etapa de abuso se observó acompañado por una recuperación (usualmente sólo parcial) basada en un mecanismo compensatorio cerebral primario de los cambios volumétricos (Pfefferbaum et al., 1995). La extensión de los cambios volumétricos se relacionó con factores temporales (Gazdzinski et al., 2005), cantidad de consumo de alcohol (Pfefferbaum et al., 1998), edad (Pfefferbaum et al., 1992) y sexo del consumidor (Hommer et al., 2001; Pfefferbaum et al., 2001).

Además de las descriptas alteraciones volumétricas a nivel de las cortezas frontal, temporal y parietal, y de las estructuras subcorticales como de la corteza cerebelosa, se destaca la injerencia del alcohol en la reducción del volumen de la protuberancia (Sullivan et al., 1996), del vermis cerebeloso (Sullivan et al., 2000) y el adelgazamiento del cuerpo calloso (Pfefferbaum et al., 1996), que también han sido reportadas.

Otro hecho considerable es la inclusión en análisis de la sustancia blanca como factor causal de desórdenes cognitivos como plantearan Pfefferbaum et al. (2000). Se generalizaron dos estudios que exploraron la relación entre el volumen de la sustancia blanca y las funciones cognitivas (Gazdzinski et al.; Sullivan et al., 2003) en alcohólicos pero con deterioro del funcionamiento social.

Hallazgos histológicos en cerebros humanos postmortem afirman la hipótesis que el área del cuerpo calloso en plano medio-sagital es una reflejo del grado de conectividad anatómica entre los hemisferios cerebrales. Existe evidencia reportada que las medidas del cuerpo calloso pueden variar acorde con las diferencias anatómicas 
hemisféricas cerebrales, y también, pueden ser relacionadas con las asimetrías hemisféricas funcionales. Estudios en ratas demostraron que el volumen del área B17 es asimétrica entre hemisferios, y dicha asimetría se traduce con correlación negativa a la densidad de las terminaciones de dicha área en el cuerpo calloso (Rosen et al., 1990). El hallazgo evidencia que la gran asimetría anatómica hemisférica se asocia con la pérdida de conectividad a nivel del cuerpo calloso. Asimismo, análogos hallazgos se han reportado en cerebros de humanos. Aboitiz et al. (1992a) hallaron que en masculinos, las asimetrías en mediciones post-mortem de la distancia entre el surco lateral (Aboitiz et al., 1992b) y el plano temporal mantenían una correlación significativamente negativa con el área en plano medio-sagital del istmo del cuerpo calloso. El istmo yace en el cuerpo posterior de la región alredeor del surco lateral. Los hallazgos de Aboitiz et al. (1992a) indican que a mayor incremento de las diferencias anatómicas de las áreas posteriores al lenguaje el tamaño de su región en el cuerpo calloso tiende a decrecer. Este hallazgo es también consistente con la hipótesis que se necesitarían mayores conexiones callosas en cerebros menos lateralizados (Witelson).

Existen datos anatómicos (de Lacoste et al.; LaMantia \& Rakic, 1990) y clínicos considerables que sugieren que el cuerpo calloso tiene una organización topográfica en relación con la funcionalidad de áreas corticales especiales. Pacientes con lesiones discretas de regiones del cuerpo callo- so sufren síndromes por desconexión importantes, por ejemplo, la desconexión visual por lesiones del esplenio posterior, la desconexión auditiva asociada con lesiones del istmo y la desconexión táctil por lesión del cuerpo del cuerpo calloso, como también la relación entre regiones anteriores con el procesamiento verbal semántico.

\section{CONCLUSIONES}

En nuestra cohorte de sujetos con trastorno por consumo de alcohol tipo dependencia, el cuerpo calloso y los cuerpos mamilares presentaron reducciones volumétricas. La superficie del cuerpo calloso con mayor reducción fue la porción del 1/3 anterior, la cual mantuvo correlación con la volumetría de los tálamos y el cuerpo amigdalino izquierdo. La reducción de los cuerpos mamilares, mayor en el hemisferio izquierdo, fue correlativa con la disminución volumétrica de los tálamos y cuerpos amigdalinos; y resultó comprometida tempranamente. Todas las estructuras cuantificadas mantuvieron un comportamiento tiempo de consumo dependiente.

Las alteraciones morfométricas evidenciadas representaron un indicador de severidad cognitiva con incidencia comportamental por su relación con el compromiso del cuerpo amigdalino.

TORNESE E. B. \& MAZZOGLIO Y NABAR, M. J. Morphometry of the corpus callosum and mammillary bodies in alcoholism using magnetic resonance Int. J. Morphol., 31(4):1233-1242, 2013.

SUMMARY: Brain morphological abnormalities in individuals with severe chronic alcoholism explains the severe neurocognitive disorders, namely neuropsychiatric and anterograde amnesia, due to the neurotoxic nature and nutritional deficits generated. The objective of the present study was to describe in alcohol dependent subjects, the morphology of the corpus callosum and mammillary bodies and volume ratio in other brain areas. We used morphological and morphometric magnetic resonance imaging to study the brains of 26 men, ages $29-51$ years diagnosed with alcohol dependence type disorder (DSM IV). Exclusion criteria were: liver diseases, autoimmune and central neurological disorders, as well as a neurological history not related to consumption, prior traumatic brain injury or brain surgery. Volumes were quantified by (ROI method) for the following: prefrontal cortex, hippocampus, amygdaloid body, thalamus and mammillary bodies, and the twodimensional morphometry of corpus callosum regions (Witelson and Clarke methods). The procedure was subject to statistical tests and current legal ethical standards. Our results showed all patients dependent volumes were lower. In accordance with severity, significant volume reduction was recorded in prefrontal cortex, mammillary body, left hippocampus and thalamus, followed by the right amygdaloid body. The most affected area of the corpus callosum was the anterior third. Correlations were determined between the involvement of the mammillary bodies and the corpus callosum with subcortical structures. The relation of the corpus callosum and mammillary bodies correlated with the involvement of the hippocampus, amygdala and thalamus body, and are closely related to the amount of time and consumption of alcohol. These morphometric changes are indicative of severe cognitive and behavioral impairment.

KEY WORDS: Morphometry; Corpus callosum; Mammillary bodies; Alcoholism.

\section{REFERENCIAS BIBLIOGRÁFICAS}

Aboitiz, F.; Scheibel, A. B.; Fisher, R.S. \& Zaidel, E. Fiber composition of the human corpus callosum. Brain Res., $598(1-$ 2):143-53, 1992a.
Aboitiz, F.; Scheibel, A. B. \& Zaidel, E. Morphometry of' the Sylvian fissure and the corpus callosum, with emphasis on sex differences. Brain, 115(Pt. 5):1521-41, 1992b. 
Bilir, E.; Craven, W.; Hugg, J.; Gilliam, F.; Martin, R.; Faught, E. \& Kuzniecky, R. Volumetric MRI of the limbic system: anatomical determinants. Neuroradiology, 40(3):138-44,1998.

Clarke, S.; Kraftsik, R.; Van der Loos, H. \& lnnocenti, G. M. Forms and measures of adult and developing human corpus callosum: Is there sexual dimorphism? J. Comp. Neurol., 280(2):213$30,1989$.

de Lacoste, M. C.; Kirkpatrick, J. B. \& Ross, E. D. Topography of the human corpus callosum. J. Neuropathol. Exp. Neurol., 44(6):578-91, 1985.

Estruch, R.; Nicolás, J. M.; Salamero, M.; Aragón, C.; Sacanella, E.; Fernández-Solà, J. \& Urbano-Márquez, A. Atrophy of the corpus callosum in chronic alcoholism. J. Neurol. Sci., 146(2):145-51, 1997.

Fein, G.; Di Sclafani, V. \& Meyerhoff, D. J. Prefrontal cortical volume reduction associated with frontal cortex function deficit in 6-week abstinent crack-cocaine dependent men. Drug Alcohol Depend., 68(1):87-93, 2002.

Gazdzinski, S.; Durazzo, T. C. \& Meyerhoff, D. J. Temporal dynamics and determinants of whole brain tissue volume changes during recovery from alcohol dependence. Drug Alcohol Depend., 78(3):263-73, 2005.

Giedd, J. N.; Rumsey, J. M.; Castellanos, F. X.; Rajapakse, J. C.; Kaysen, D.; Vaituzis, A. C.; Vauss, Y. C.; Hamburger, S. D. \& Rapoport, J. L. A quantitative MRI study of the corpus callosum in children and adolescents. Brain Res. Dev. Brain Res., 91(2):274-80, 1996.

Hommer, D.; Momenan, R.; Rawlings, R.; Ragan, P.; Williams, W.; Rio, D. \& Eckardt, M. Decreased corpus callosum size among alcoholic women. Arch. Neurol., 53(4):359-63, 1996.

Hommer, D.; Momenan, R.; Kaiser, E. \& Rawlings, R. Evidence for a gender-related effect of alcoholism on brain volumes. Am. J. Psychiatry, 158(2):198-204, 2001.

Iai, M.; Tanabe, Y.; Goto, M.; Sugita, K. \& Niimi, H. A comparative MRI study of the corpus callosum in neurologically normal children and children with spastic diplegia. Acta Paediatr., 83(10):1086-90, 1994.

Laissy, J. P.; Patrux, B.; Duchateau, C.; Hannequin, D.; Hugonet, P.; Ait-Yahia, H. \& Thiebot, J. Midsagittal MR measurements of the corpus callosum in healthy subjects and diseased patients: a prospective survey. AJNR Am. J. Neuroradiol., 14(1):145$54,1993$.

LaMantia, A. S. \& Rakic, P. Cytological and quantitative characteristics of four cerebral commissures in the rhesus monkey. J. Comp. Neurol., 291(4):520-37,1990.

Pfefferbaum, A.; Sullivan, E. V.; Hedehus, M.; Adalsteinsson, E.; Lim, K. O. \& Moseley, M. In vivo detection and functional correlates of white matter microstructural disruption in chronic alcoholism. Alcohol. Clin. Exp. Res., 24(8):1214-21, 2000.

Pfefferbaum, A.; Lim, K. O.; Zipursky, R. B.; Mathalon, D. H.; Rosenbloom, M. J.; Lane, B.; Ha, C. N. \& Sullivan, E. V. Brain gray and white matter volume loss accelerates with aging in chronic alcoholics: A quantitative MRI study. Alcohol. Clin. Exp. Res., 16(6):1078-89, 1992.

Pfefferbaum, A.; Sullivan, E. V.; Mathalon, D. H.; Shear, P. K.; Rosenbloom, M. J. \& Lim, K. O. Longitudinal changes in magnetic resonance imaging brain volumes in abstinent and relapsed alcoholics. Alcohol. Clin. Exp. Res., 19(5):1177-91, 1995.

Pfefferbaum, A.; Lim, K. O.; Desmond, J. \& Sullivan, E. V. Thinning of the corpus callosum in older alcoholic men: A magnetic resonance imaging study. Alcohol. Clin. Exp. Res., 20(4):752-7, 1996.

Pfefferbaum, A. \& Sullivan, E. V. Microstructural but not macrostructural disruption of white matter in women with chronic alcoholism. Neuroimage, 15(3):708-18, 2002.

Pfefferbaum, A.; Sullivan, E. V.; Mathalon, D. H. \& Lim, K. O. Frontal lobe volume loss observed with magnetic resonance imaging in older chronic alcoholics. Alcohol. Clin. Exp. Res., 21(3):521-9, 1997.

Pfefferbaum, A.; Sullivan, E. V.; Rosenbloom, M. J.; Mathalon, D. H. \& Lim, K. O. A controlled study of cortical gray matter and ventricular changes in alcoholic men over a five-year interval. Arch. Gen. Psychiatry, 55(10):905-12, 1998.

Pfefferbaum, A.; Rosenbloom, M. J.; Deshmukh, A. \& Sullivan, E. V. Sex differences in the effects of alcohol on brain structure. Am. J. Psychiatry, 158(2):188-97, 2001.

Pruessner, J. C.; Li, L. M.; Serles, W.; Pruessner, M.; Collins, D. L.; Kabani, N.; Lupien, S. \& Evans, A. C. Volumetry of hippocampus and amygdala with high resolution MRI and three-dimensional analysis software: minimizing the discrepancies between laboratories. Cereb. Cortex, 10(4):43342, 2000 .

Rosen, G. D.; Galaburda, A. M. \& Sherman, G. F. The ontogeny of anatomic asymmetry: constraints derived from basic mechanisms. In: Scheibel, A. B. \& Wechsler, A. F. (Eds.). Neurobiology of Higher Cognitive Function. New York, Guilford, 1990. pp.215-38.

Sullivan, E. V.; Marsh, L.; Mathalon, D. H.; Lim, K. O. \& Pfefferbaum, A. Relationship between alcohol withdrawal seizures and temporal lobe white matter volume deficits. Alcohol. Clin. Exp. Res., 20(2):348-54, 1996.

Sullivan, E. V. Human brain vulnerability to alcoholism: Evidence from neuroimaging studies. In: Noronha, A.; Eckardt, M. \& Warren, K. (Eds.). Review of NIAAA's Neuroscience and 
Behavioral Research Portfolio. NIAAA Research Monograph No. 34. Bethesda, National Institutes of Health, 2000. pp.473508 .

Sullivan, E. V.; Rosenbloom, M. J.; Serventi, K. L.; Deshmukh, A. $\&$ Pfefferbaum, A. The effects of alcoholism-schizophrenia comorbidity on volumes of the thalamus and pons. Am. J. Psychiatry, 160(6):11106, 2003.

Verger, K.; Junqué, C.; Levin, H. S.; Jurado, M. A.; Pérez-Gómez, M.; Bartrés-Faz, D.; Barrios, M.; Alvarez, A.; Bartumeus, F. \& Mercader, J. M. Correlation of atrophy measures on MRI with neuropsychological sequelae in children and adolescents with traumatic brain injury. Brain Inj., 15(3):211-21, 2001.

Wible, C. G.; Shenton, M. E.; Hokama, H.; Kinikis, R.; Josesz, F. A.; Metcalf, D. \& McCarley, R. W. Prefrontal cortex and schizophrenia: a quantitative magnetic resonance imaging study. Arch. Gen. Psychiatry, 52(4):279-88,1995.

Witelson, S. F. Hand and sex differences in the isthmus and genu of the human corpus callosum. Brain, 112(Pt. 3):799-835,1989.

Zaidel, E.; Aboitiz, F. \& Clarke, J. Sexual dimorphism in interhemispheric relations: anatomical-behavioral convergence. Biol. Res., 28(1):27-43, 1995.

\author{
Dirección para Correspondencia: \\ Martín Javier Mazzoglio y Nabar \\ Facultad de Medicina \\ Universidad Nacional de Buenos Aires \\ Paraguay, 2055, 4o piso, Sector Uriburu \\ CP 1121 \\ Buenos Aires \\ ARGENTINA
}

Email: mazzoglioynabar@hotmail.com

Recibido : 19-05-2013

Acceptado: 10-08-2013 